\title{
Acute Acalculous Cholecystitis as a Presenting Manifestation in Systemic Lupus Erythematosus
}

\author{
Sonmoon Mohapatra ${ }^{1}$, Debra R Goldstein ${ }^{2}$, Akshat Kumar ${ }^{1}$, Trisha Saha ${ }^{1}$, Damodar Penigalapati ${ }^{1}$ \\ ${ }^{1}$ Department of Internal Medicine, Rutgers Robert Wood Johnson Medical School /Saint Peter's University Hospital, New Brunswick, New Jersey, USA \\ ${ }^{2}$ Department of Gastroenterology and Hepatology, Rutgers Robert Wood Johnson Medical School/Saint Peter's University Hospital, New Brunswick, \\ New Jersey, USA
}

\section{Doi: 10.12890/2016_000408 - European Journal of Case Reports in Internal Medicine - ๑ EFIM 2016}

Received: $18 / 02 / 2016$

Accepted: $22 / 03 / 2016$

Published: $11 / 05 / 2016$

How to cite this article: Mohapatra S, Goldstein DR, Kumar A, Saha T, Penigalapati D. Acute acalculous cholecystitis as a presenting manifestation in systemic lupus erytematosus. EJCRIM 2016;3:doi:10.12890/2016_000408.

Conflicts of Interests: The Authors declare that there are no competing interests.

This article is licensed under a Commons Attribution Non-Commercial 4.0 License

\section{ABSTRACT}

Systemic lupus erythematosus (SLE) is known to involve the gastrointestinal tract, but gallbladder involvement is rare. The authors report the case of a 26-year-old postpartum female who presented with acute right upper quadrant abdominal pain and was diagnosed with acute acalculous cholecystitis (AAC). In the presence of concomitant features of nephritis, pericardial effusion, anaemia and positive ANA titre, the diagnosis of SLE was confirmed during hospitalisation. Histopathological analysis of the gall bladder revealed evidence of vasculitis. Although rare, AAC can be the first presentation of patients diagnosed with SLE. Prompt diagnosis and management results in a better patient outcome.

\section{LEARNING POINTS}

- Although rare, acute acalculous cholecystitis is to be suspected in patients in the setting of right upper quadrant pain associated with multisystem disease as it may manifest as an initial symptom of the multisystem disease.

- In women with pre-existing rheumatological disease, a high index of suspicion for manifestations of postpartum flares should be maintained.

- Definitive treatment includes cholecystectomy; high-dose steroid therapy has been reported to be successful in a few cases.

\section{KEYWORDS}

Systemic lupus erythematosus, acalculous cholecystitis, gallbladder vasculitis.

\section{INTRODUCTION}

Systemic lupus erythematosus (SLE) is a systemic autoimmune inflammatory disease with a wide spectrum of clinical manifestations. SLE seldom presents as acute acalculous cholecystitis (AAC). Initial presentation of SLE as AAC is also rare: only four cases have been reported in the literature ${ }^{[1,2]}$. This is the first reported case of SLE manifested as AAC during the postpartum period.

\section{CASE REPORT}

A 26-year-old Caucasian female presented with acute right upper quadrant (RUQ) pain. Two weeks previously she had had a caesarean section performed emergently at 28 weeks for what was thought to be severe pre-eclampsia. At the time of presentation, her vital signs were temperature $98.4^{\circ} \mathrm{F}, \mathrm{BP} 119 / 81 \mathrm{mmHg}, \mathrm{HR} 104 / \mathrm{min}, \mathrm{RR} 20 / \mathrm{min}$, and $\mathrm{O}_{2}$ saturation $100 \%$ on $2 \mathrm{~L}$ nasal cannula. Physical examination showed 
pallor, decreased breath sounds at the lung bases, RUQ tenderness with positive Murphy's sign and bilateral pedal oedema. Laboratory

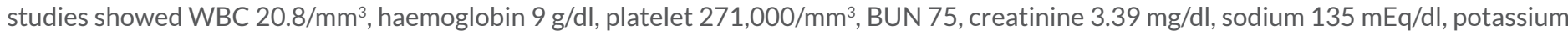
$5.1 \mathrm{mEq} / \mathrm{dl}$, chloride $105 \mathrm{mEq} / \mathrm{dl}$, bicarbonate $18 \mathrm{mEq} / \mathrm{dl}$, total bilirubin $0.6 \mathrm{mg} / \mathrm{dl}$, AST $38 \mathrm{IU} / \mathrm{l}$, ALT $25 \mathrm{IU} / \mathrm{l}$, alkaline phosphatase $161 \mathrm{IU} / \mathrm{l}$ and ESR $97 \mathrm{~mm} / \mathrm{hr}$. Amylase and lipase level were normal. Urine analysis was positive for haematuria and 3+ proteinuria. Abdominal ultrasound showed gallbladder wall thickening and a normal sized common bile duct without evidence of stones. A clinical diagnosis of acute cholecystitis was made. Hepatobiliary iminodiacetic acid (HIDA) scan was unremarkable. The patient was empirically started on antibiotics. During hospitalization the RUQ pain worsened and the patient developed associated nausea, vomiting and fever. In the setting of the persistent RUQ pain and positive Murphy's sign, a cholecystectomy was performed. Histology of the gallbladder specimen revealed focal areas of acute inflammation and fibrinoid changes suggestive of small vessel vasculitis (Fig. 1). Given the patient's complex clinical presentation and multi-organ involvement, the diagnosis of SLE was considered. Immunological testing showed positive ANA (speckled pattern 1:1280), C3 $80 \mathrm{mg} / \mathrm{dl}$, and C4 <8 mg/dl. Anti-dsDNA, lupus anticoagulant, anti-Smith antibody and anti-B2-glycoprotein antibody were all negative. The renal biopsy (Fig.1) showed diffuse endocapillary proliferative glomerulonephritis, immune complex type, suggestive of lupus nephritis (ISNRPS class IV-G). The patient's condition markedly improved after surgery and she was discharged with oral prednisone and mycophenolate.

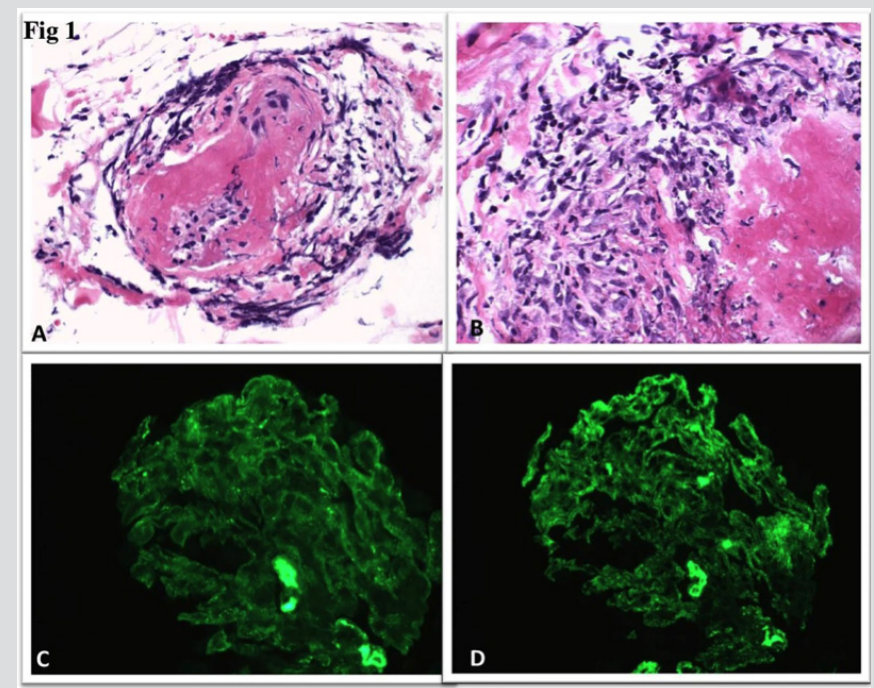

Figure 1 (A,B). Microscopic appearance of the gallbladder showing acute arteritis with fibrinoid changes and degeneration of the media of the arteries. $(C, D)$ : Immunofluorescence finding of diffuse granular mesangial and glomerular capillary wall staining for $\lg \mathrm{G}, \lg \mathrm{M}$ and $\mathrm{C} 3$, supporting the diagnosis of an immune-complex mediated glomerulonephritis such as lupus nephritis.

\section{DISCUSSION}

Gastrointestinal (GI) manifestations of SLE include mouth ulcers, dysphagia, anorexia, nausea, vomiting, haemorrhage and abdominal pain. Symptoms can develop either from autoimmune tissue injury, complications of lupus-related organ dysfunction, thromboembolic manifestations of anti-phospholipid antibody syndrome, infection, medication and unrelated disorders. While the most common GI manifestations of SLE are seen in the oral cavity, GI vasculitis may potentially contribute to greater morbidity and mortality. Early recognition and treatment is important to optimize long-term survival.

GI manifestations of lupus vasculitis are abdominal pain, nausea and vomiting. Although non-specific, these are usually accompanied by evidence of active disease elsewhere affecting the skin, renal and cardiovascular systems. The classic histological feature of cholecystitis in SLE is vasculitis of small vessel arteries and venules. Associated findings may include atrophy and degeneration of the media of small arteries, fibrinoid necrosis of the vessel walls, old thrombosis, phlebitis and monocyte infiltrate in the lamina propria ${ }^{[2]}$. Although vasculitis is a common complication of SLE, manifesting in up to $18 \%$ of patients, it is usually a cutaneous disease ${ }^{[3]}$. In this case, it was concluded that the cholecystitis was the result of vasculitis due to SLE.

Helpful diagnostic parameters include complete blood count, liver and renal function tests, lactate, ANA, anti-dsDNA, C-reactive protein, blood sedimentation, C3 and C4 complement levels and antiphospholipid antibody status. Abdominal ultrasound, hepatobiliary scintigraphy and CT scan are indicated for the diagnosis. HIDA scan may be normal in $\mathrm{ACC}^{[4]}$. The most characteristic radiological findings, increased gall 
bladder wall thickness and accumulated fluid in the gall bladder fossa in the absence of gallstones or sludge, were present in our imaging studies.

AAC is known to have a high mortality rate. Definitive treatment includes cholecystectomy. In patients who are poor surgical candidates, cholecystostomy remains another option. Endoscopic therapy with nasobiliary drainage is a third option for patients who cannot tolerate either surgery or cholecystostomy ${ }^{[5]}$. High-dose steroid therapy has been reported to be successful in a few cases and can be tried in selected patients, depending on general condition, disease severity and associated risk factors ${ }^{[1,2]}$.

This case posed a diagnostic challenge. The patient symptoms satisfied the American College of Rheumatology (ACR) classification criteria of SLE. She had abdominal pain as well as renal failure and pericardial effusion. Anti-dsDNA antibody was negative despite the presence of active disease. SLE is well known for its atypical presentations, and this patient had a rare manifestation of SLE. As she had undergone caesarean section for suspected pre-eclampsia 2 weeks previously, we suspected acute cholecystitis secondary to gallstone disease. On further evaluation, however, the patient was found to have AAC secondary to SLE, which itself is often seen to flare in the immediate postpartum period.

Our patient, managed promptly, markedly improved after surgery. Our case is the first reported case of SLE manifested as AAC during the postpartum period. Although rare, we want to emphasize that AAC can present as an initial manifestation of SLE. Prompt diagnosis and management is required for better patient outcome.

\section{REFERENCES}

1. Manuel V, Pedro GM, Cordeiro LB, Miranda SM. Acute acalculous cholecystitis in systemic lupus erythematosus: a rare initial manifestation. Rev Bras Reumatol (English Edition) 2014. DOI:10.1016/j.rbre.2014.03.027.

2. Yun Jung Choi HYY, Seol A Jang, Myong Joo Hong, Won Seok Lee, Wan-Hee Yoo. A case of systemic lupus erythematosus initially presented with acute acalculous cholecystitis J Rheum Dis 2014;21:140-142.

3. Hegarty J, Chiu DY, Middleton RJ, Haeney MM, Newstead CG, Bruce IN, et al. Systemic lupus erythematosus, eosinophilic vasculitis and acalculous cholecystitis. Nephrol Dial Transplant 2006;21:792-795.

4. Montini KM, Tulchinsky M. Applied hepatobiliary scintigraphy in acute cholecystitis. Appl Radiol 2015;44:22-30.

5. Owen CC, Jain R. Acute acalculous cholecystitis. Curr Treat Options Gastroenterol 2005;8:99-104. 